\title{
O funículo umbilical em bovinos azebuados
}

\author{
The funiculus umbilicalis in crossbred zebu bovines
}

\author{
Antonio Augusto Coppi Maciel RIBEIRO'; Maria Angelica MIGLINO²
}

CORRESPONDENCE TO:

Antonio Augusto Coppi Maciel

Ribeiro

Departamento de Anatomia

Instituto de Biociências da UNESP

18618-000 - Botucatu - SP

$18618-0$

Brasil

e-mail: aaugusto@ibb.unesp.br

1 - Departamento de Anatomia

Instituto de Biociências da

UNESP - Botucatu - SP

2 - Departamento de Cirurgia

Faculdade de Medicina

Veterinária e Zootecnia da USP

\section{RESUMO}

Nesta pesquisa, obtiveram-se dados sobre os aspectos microscópicos das estruturas vasculares que compõem o funículo umbilical de bovinos azebuados. Para a realização da pesquisa utilizaram-se funículos umbilicais provenientes de 30 fetos (13 machos e 17 fêmeas).Tanto as artérias quanto as veias apresentavam, na constituição das suas paredes, um repregueamento da túnica íntima que se mostrava bastante característico nas artérias. A túnica média das artérias e veias continha uma musculatura bem desenvolvida, fato que caracterizava esses vasos do tipo muscular. A luz dos vasos arteriais e venosos mostrava-se inicialmente (porção justa-fetal) quase obliterada e aumentava à medida que se aproximava da porção justa-placentária do funículo umbilical. A quantidade de fibras reticulares, elásticas e colágenas na parede dos vasos umbilicais foi comparada mediante técnicas histológicas específicas. Alguns aspectos inerentes ao ducto alantóide foram também observados.

UNITERMOS: Funículo umbilical; Bovinos; Artérias; Veias.

\section{INTRODUÇÃO}

$\mathrm{E}$ sta pesquisa tem por objetivo obter dados sobre os aspectos microscópicos a respeito dos elementos vasculares que compõem o funículo umbilical de bovinos azebuados. Por meio de estudo detalhado sobre a estrutura da parede dos vasos umbilicais, analisamos a constituição anatômica das artérias e veias, estabelecendo a análise comparativa entre elas nos diferentes segmentos do funículo umbilical.

A escassez de dados bibliográficos sobre bovinos de origem indiana e a oportunidade de comparar nossos achados com resultados de trabalhos semelhantes realizados em bovinos das raças Holstein e Hereford, de origem européia, constituíram incentivos importantes para a execução desta investigação científica.

\section{MATERIAL E MÉTODO}

Para a realização deste trabalho obtivemos funículos umbilicais de 30 fetos bovinos azebuados.

Foram realizados cortes transversais nas porções justa-fetal, intermediária e justa-placentária do funículo umbilical para serem submetidos a diferentes técnicas histológicas, com o intento de estudar a estrutura e disposição desse funículo.

A fixação dos fragmentos do funículo umbilical foi feita em solução aquosa de formol a $10 \%$ durante 72 horas, seguida dos procedimentos normais para a realização de cortes histológicos corados pelas técnicas da Hematoxilina-Eosina (H.E.); Mallory, Orceína, Reticulina, Picrossírius sem polarização e Picrossírius com polarização, esta última proposta por Junqueira el al. ${ }^{3}$ (1979).

O Picrossírius, marcado com a microscopia polarizada, constitui-se numa técnica específica para detecção do colágeno em cortes de tecidos. O colágeno, sendo uma proteína básica, reage com os grupos sulfônicos presentes no corante sirius red, de tal modo que as moléculas do corante ficam paralelas às fibras colágenas, resultando uma acentuada birrefringência, pois o tecido absorve a luz do microscópio e reflete a cor, que depende da concentração do corante por unidade da área. Portanto, para o colágeno do tipo l, as fibras em escala crescente de espessura coram-se em: amarelo, alaranjado e vermelho. Já as fibras colágenas do tipo 3 (fibras reticulares), sendo mais finas, coram-se em verde.

Dos preparados foram tiradas fotografias com auxílio do fotomicroscópio $\mathrm{BH}-2$ Olimpus, pertencente ao Departamento de Patologia Animal da Faculdade de Medicina Veterinária e Zootecnia da Universidade de São Paulo. Essas fotografias apresentaram aumento de $20,50,66,127,165$ e 660 vezes.

\section{RESULTADOS}

Tanto as artérias quanto as veias apresentaram, na constituição das suas paredes, uma túnica interna ou íntima, que mostrava um repregueamento mais característico nas artérias, uma túnica média, que continha uma musculatura bem desenvolvida, e uma 
túnica adventícia. Os vasos umbilicais eram do tipo muscular e apresentavam espessa túnica média (veias e artérias). Sua luz inicialmente pequena ou quase obliterada aumentava à medida que se aproximava da porção justa-placentária do funículo umbilical (Fig. I).

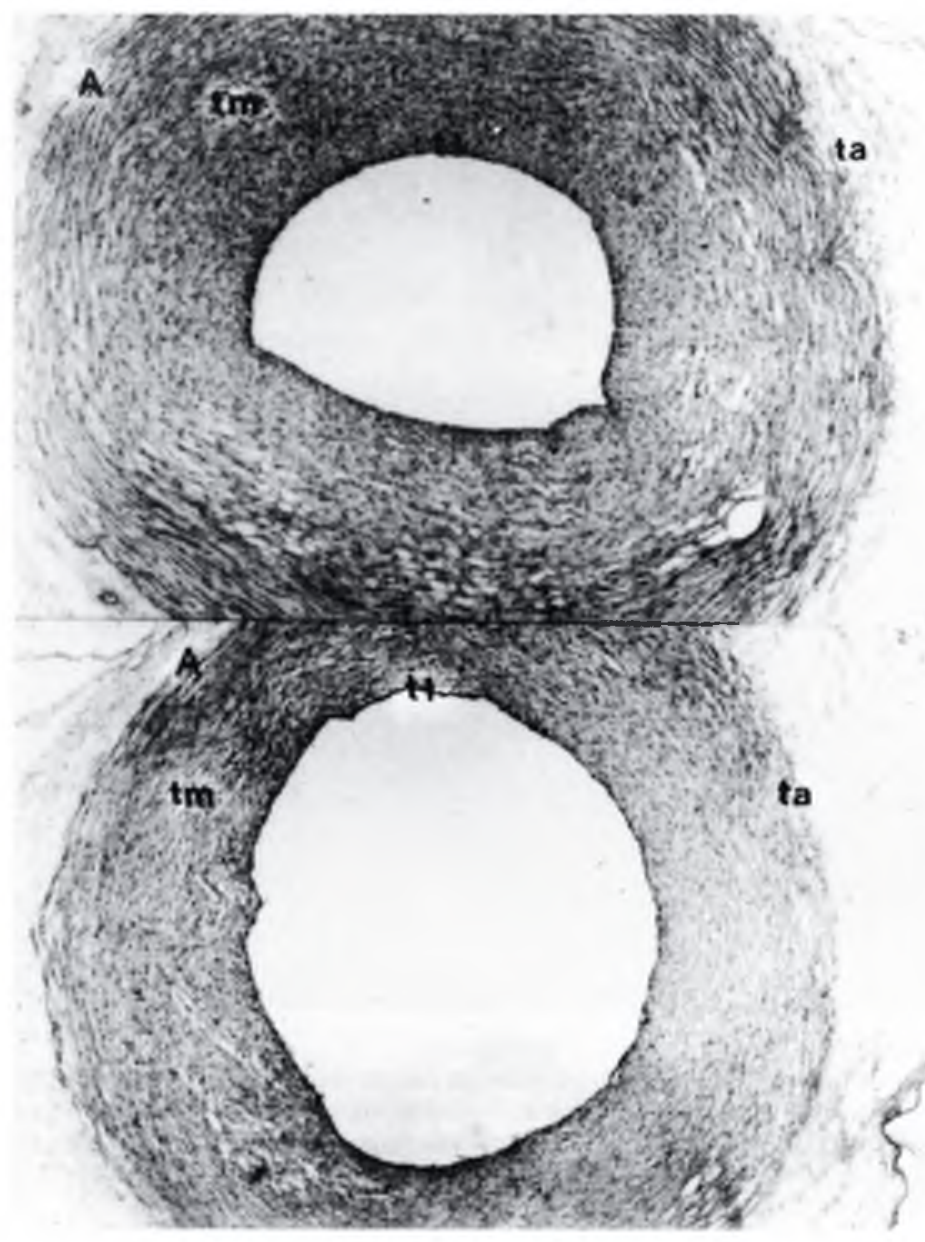

Figura1

Fotomicrografia da seccão transversal de uma artéria umbilical (A) mostrando o repregueamento da sua túnica íntima (ti), uma túnica média (tm) bem desenvolvida e uma túnica adventícia (ta). Nota-se a ampliação da luz da artéria à medida que se aproxima da porção justa-placentária do funículo umbilical. Hematoxilina-Eosina. Aumento: 66x.

A luz das veias caracterizava-se por apresentar aspecto estrelado (Fig. 2) ou quase obliterado na porção justa-fetal do funículo umbilical.

Fibras reticulares eram características na parede dos vasos umbilicais (artérias e veias), embora fossem mais numerosas e evidentes na parede das veias umbilicais (Fig. 3).

Fibras elásticas curtas, finas e delicadas foram também evidenciadas em pequena quantidade nas paredes das artérias e veias umbilicais ( Fig. 4).

As fibras colágenas que compõem a estrutura da parede dos vasos umbilicais apresentaram disposições variadas e espessuras diferentes.

As veias umbilicais são mais ricas em fibras colágenas, principalmente na sua túnica adventícia, em relação às artérias, pois

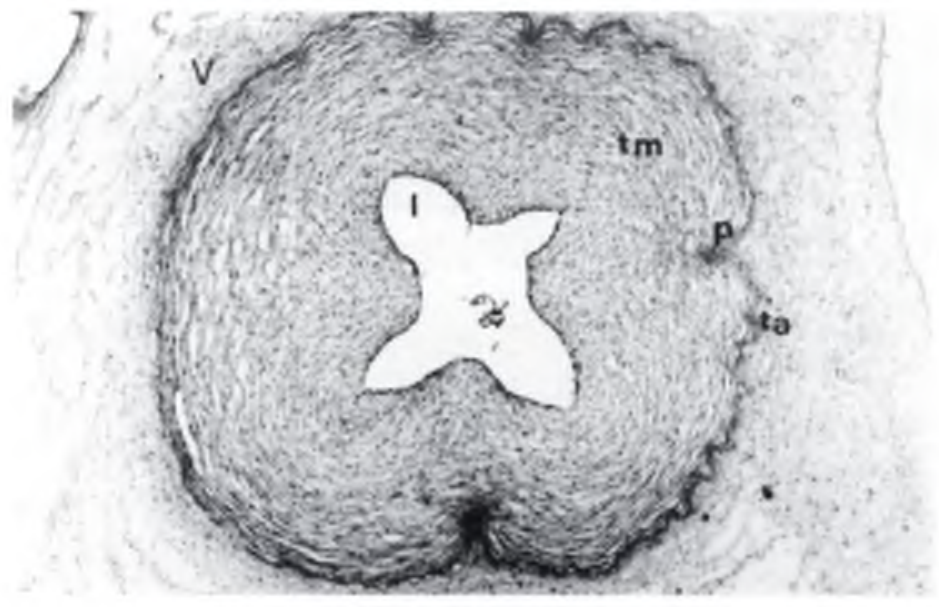

Figura 2

Fotomicrografia da secção transversal de uma veia umbilical (V) na porção justa-fetal do funículo umbilical, mostrando a sua luz (I) de aspecto estrelado. Sua túnica média (tm) è bem desenvolvida à semelhança do que ocorre nas artérias, porém sua túnica adventicia (ta) apresenta projeções (p) em direção à túnica média $(\mathrm{tm})$. Hematoxilina-Eosina. Aumento: $66 \mathrm{x}$

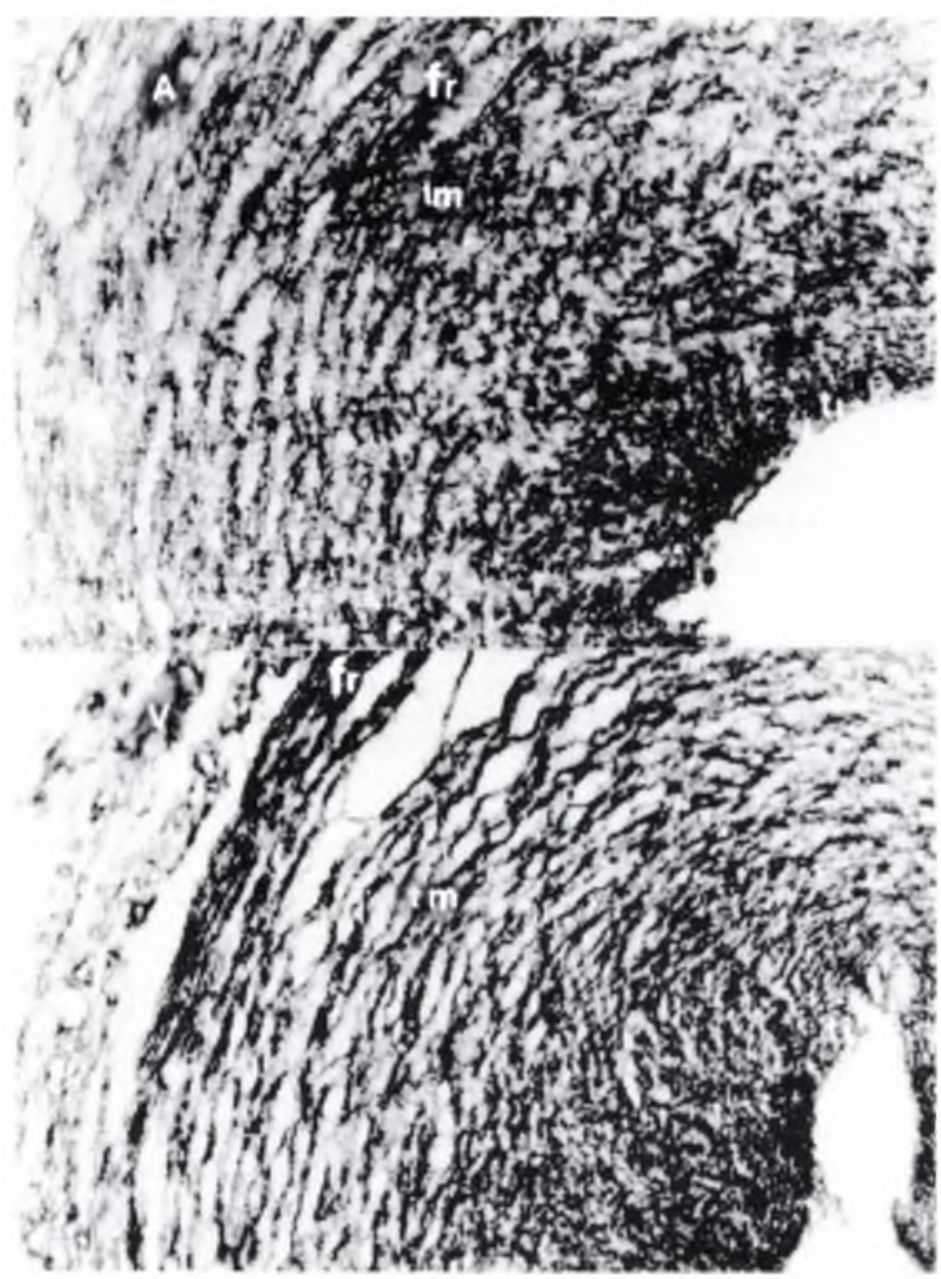

Figura 3

Fotomicrografia da secção transversal de uma artéria $(A)$ e de uma veia umbilical (V), onde se nota a presença de fibras reticulares (fr) distribuidas na túnica média (tm) e túnica íntima (ti) desses vasos.

Reticulina. Aumento: 165x. 
essas fibras distribuem-se de maneira mais condensada.

Por meio da técnica histológica denominada Picrossírius com polarização, evidenciamos a presença de fibras colágenas de diferentes espessuras e colorações, em que as fibras vermelhas, alaranjadas ou amarelas foram classificadas como colágeno do tipo 1 e as demais libras verdes, como colágeno do tipo 3 (Fig. 5).

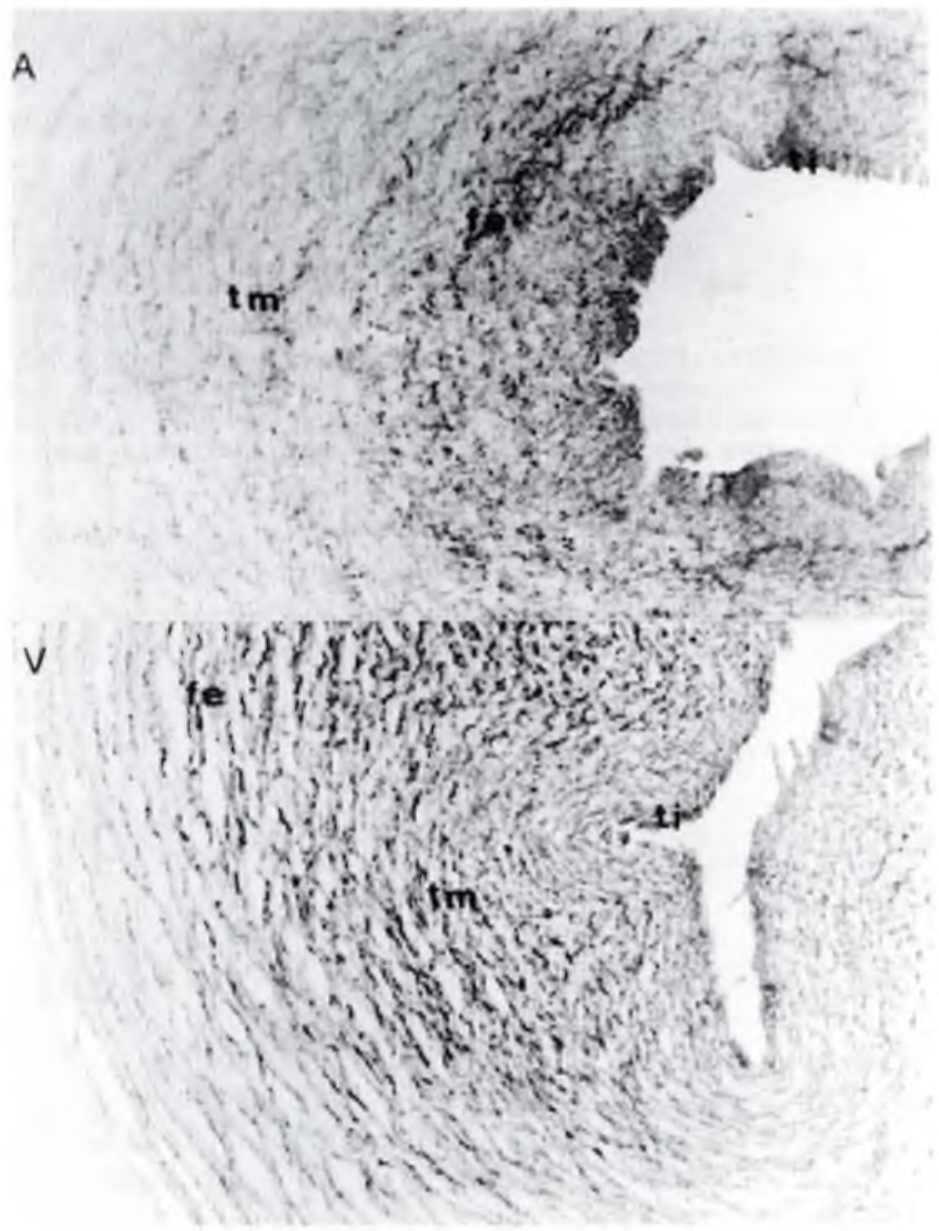

Figura 4

Fotomicrografia da secção transversal de uma artéria (A) e veia umbilical (V), onde se vêem as fibras elásticas (fe) distribuídas na túnica média (tm) e túnica intima (ti) desses vasos, coradas em marrom-escuro. Orceína. Aumento: 165x

\section{DISCUSSÃO}

Com relação aos aspectos histológicos ou microscópicos das paredes dos vasos do funículo umbilical de bovinos, constatamos que há carência de informações na literatura sobre este estudo, destacando-se somente Miglino et al..$^{5}$ (1994), que fizeram menção a estes aspectos ao estudarem as secções transversais de diferentes porções do funículo umbilical, ou seja, as porções justa-fetal, intermediária e justa-placentária.

Porém, no tocante às técnicas histológicas, os autores citados utilizaram somente a Hematoxilina-Eosina (H.E.), enquanto nós nos valemos de outras, como por exemplo: Orceína, Reticulina, Mallory, Picrossírius com polarização e Picrossírius sem polarização, com o intuito de descrever todos os pormenores da estrutura

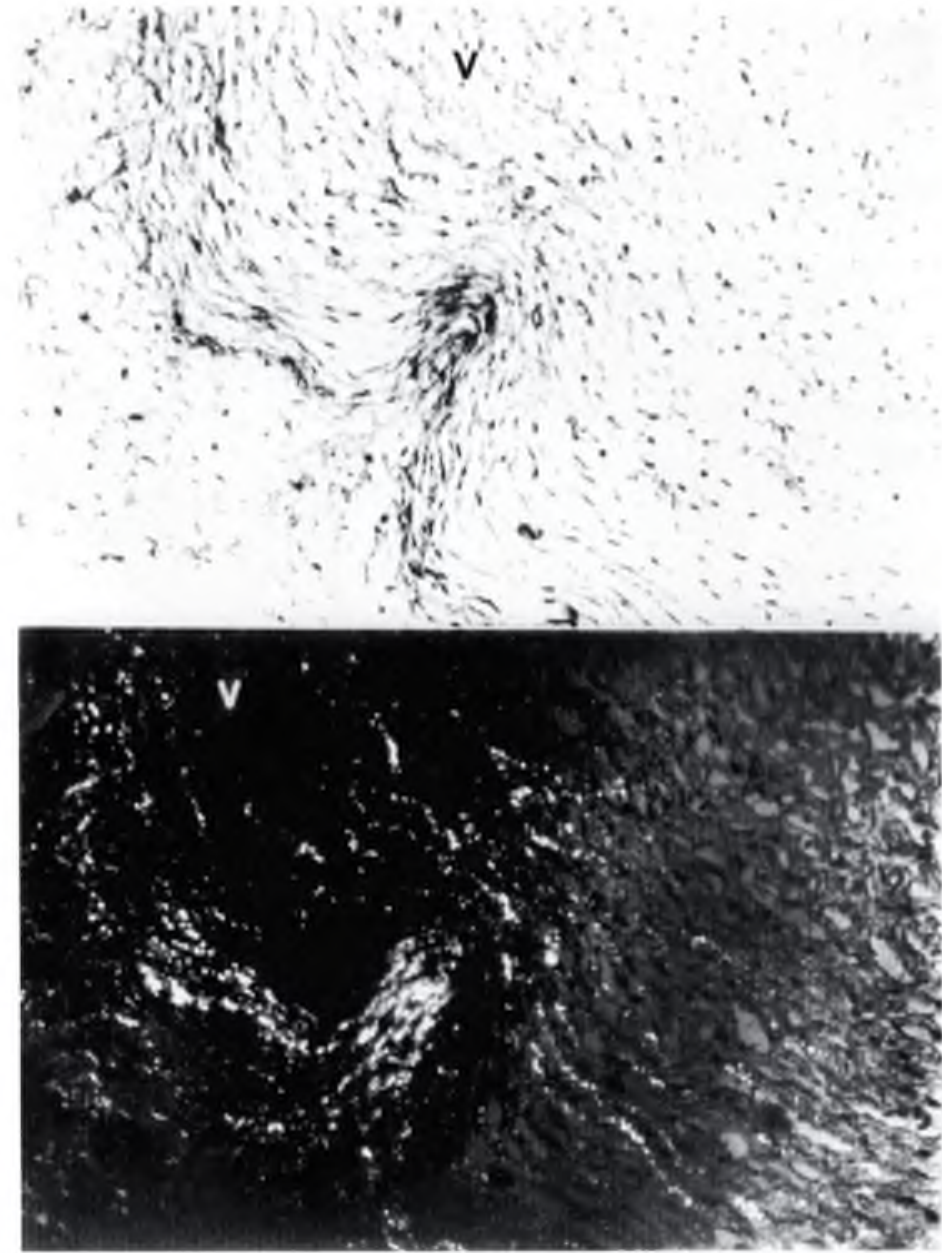

Figura 5

Fotomicrografia da seccão transversal da parede de uma veia umbilical (V) $\mathrm{na}$ porção justa-placentária do funículo umbilical, mostrando o arranjo das fibras colágenas na estrutura de sua parede e as colorações dessas fibras: vermelhas, alaranjadas, amarelas e verdes. Picrossírius sem polarização e Picrossírius com polarização. Aumento: $50 x$.

parietal dos referidos vasos, que não seriam tão amplamente revelados somente pela técnica da Hematoxilina-Eosina (H.E.).

Ressaltamos que, durante a realização deste trabalho, recorremos à última edição do International Committee on Veterinary Gross Anatomical (1994), para obter subsídios em relação à correta nomenclatura de alguns termos empregados nesta pesquisa.

Ratificando os achados de Marshall ${ }^{+}$(1952), Barone' (1986) e Miglino et $a l .5$ (1994), observamos que os vasos umbilicais são do tipo muscular, apresentando espessa túnica média, característica tanto das veias quanto das artérias.

Para as artérias, encontramos o formato da luz muito diversificado, variando de circular ou irregular até ovalado e alongado, conforme a porção do funículo umbilical considerada, fato esse que se soma aos relatos de Miglino et al..$^{5}$ (1994) em taurinos. De outra parte, a luz das veias caracterizou-se por apresentar aspecto poligonal até em "H", fato não caracterizado nas outras pesquisas sobre o mesmo assunto.

No que se refere à estrutura parietal dos vasos umbilicais, como, por exemplo, as fibras reticulares, colágenas e elásticas identificadas por algumas técnicas mais específicas, pudemos verificar que não se constituíram em objeto de estudo de outros autores 
consultados, portanto estes resultados não podem ser comparados nesta oportunidade, porém representam a nossa contribuição para este assunto e propostas para investigações futuras.

\section{CONCLUSÕES}

Do presente estudo, pode-se concluir que:

1) Tanto as artérias quanto as veias umbilicais apresentavam, na constituição das suas túnicas íntimas, um repregueamento (mais característico nas artérias);

2) Os vasos umbilicais eram do tipo muscular, apresentando uma espessa túnica média, tanto nas veias quanto nas artérias Sua luz, inicialmente pequena ou quase obliterada, aumentava à medida que se aproximava da porção justa-placentária do funículo umbilical:

3) A luz das veias caracterizava-se por apresentar aspecto estrelado ou quase obliterado na porção justa-fetal do funículo umbilical; poligonal na porção intermediária; e em " $\mathrm{H}$ " na porção justa-placentária do funículo;

4) Nas artérias, o formato da luz era circular ou irregular na porção justa-fetal do funículo umbilical, ovalado na porção intermediária e apresentava um alongamento lateral bem evidente na porção justa-placentária do funículo umbilical;
5) Fibras reticulares eram características na parede dos vasos umbilicais (artérias e veias), embora fossem mais numerosas e evidentes na parede das veias umbilicais;

6) Fibras elásticas curtas, finas e delicadas foram também evidenciadas em pequena quantidade nas paredes das artérias e veias umbilicais;

7) Em relação às veias umbilicais, pôde-se constatar que eram ricas em fibras colágenas, principalmente na sua túnica externa ou adventícia;

8) Na estrutura da parede das veias umbilicais ocorriam, de forma freqüente, fïbras colágenas, pertencentes à lúnica externa, em direção à túnica média;

9) Nos locais das vaginações nota-se a presença de fibras colágenas de diferentes colorações, em que as fibras coradas em vermelho, alaranjado e amarelo foram classificadas como colágeno do tipo I, e as fibras coradas em verde, como colágeno do tipo 3;

10) Nas artérias umbilicais, a distribuição das fibras colágenas ocorreu de maneira mais esparsa, menos concentrada, como nas veias umbilicais. As fibras colágenas eram dos tipos 1 e 3, destacando-se as coloraçōes amarelo e verde.

\section{SUMMARY}

In this study information about the microscopic aspects of the structures that form the funiculus umbilicalis of zebu like cattle was collected. For this purpose, it was used funiculus umbilicalis of 30 fetuses (13 males, 17 females). Both arteries and veins showed wrinkles in the intima layer in their wall constitution, what was more characteristic in the arteries. The medium layer of arteries and veins showed well developed muscle, characterizing these vessels as muscular type ones. At the initial portion (juxta-fetal portion) the lumen of arteries and veins was almost obliterated, but it enlarged as they got closer to the juxta-placentaria portion of the funiculus umbilicalis. The amount of reticular, elastic and collagen fibers on the vessel wall was compared by the use of specific histological techniques. Some aspects about the alantoid duct were also noticed.

\section{UNITERMS: Funiculus umbilicalis; Bovine; Arteries; Veins.}

\section{REFERÊNCIAS BIBLIOGRÁFICAS}

1 - BARONE, R. Anatomie comparée des mammiferes domestiques. Splanchnologia, Paris, Ed. Vigol, T.3, p.579-605, 1986.

2 - INTERNATIONAI COMMITTEE ON VETERINARY GROSS ANATOMICAI. Nomina anatomica, histologica, emhriologica veterinaria. 4. ed. Zurich. World Association of Veterinary Anatomists, p. 304, 1994.
3 - JUNQUEIRA, I.C.U.; BIGNOLAS, G.; BRENTANI, R.R. Picrossirius staining plus polarization microscopy, a specilic method lor collagen detection in tissuc sections. Histochemical Journal, v. II, n. 1, p.447-55, 1979.

4 - MARSHAI,I, F.H.A. Physiology of reproduction, I ondon, I ongmans, 1952. p. 189-211.

5 - MIGLINO, M.A.; DIIDIO, L.J.A.; TEOHII OVSKI-PARAPII), (i. Allantoid duct, arterius and veins of the funiculus umbilicalis in bovines. Revista Chilena de Anatomia. v. 12, n. 1, p. 61-4, 1994. 